\title{
ERS International Congress 2017: a peek of outstanding abstracts from the clinical assembly
}

\author{
Nicolas Kahn ${ }^{1,2}$, Vasileios Andrianopoulos ${ }^{3}$, Evgeni Mekov ${ }^{4}$, Felix J. F. Herth ${ }^{1,2}$ \\ ${ }^{1}$ Department of Pneumology and Critical Care Medicine, Thoraxklinik, Heidelberg University Hospital, Heidelberg, Germany; ${ }^{2}$ Translational Lung \\ Research Center Heidelberg (TLRC), Member of the German Center for Lung Research (DZL), Heidelberg, Germany; ${ }^{3}$ Department of Respiratory \\ Medicine \& Pulmonary Rehabilitation, Schoen Klinik Berchtesgadener Land, Schoenau am Koenigssee, Germany; ${ }^{4}$ Medical University—Sofia, \\ Medical Faculty, Clinical Center for Pulmonary Diseases, MHATPD 'Sveta Sofia', Sofia, Bulgaria \\ Contributions: (I) Conception and design: N Kahn; (II) Administrative support: FJF Herth; (III) Provision of study materials or patients: All authors; \\ (IV) Collection and assembly of data: All authors; (V) Data analysis and interpretation: All authors; (VI) Manuscript writing: All authors; (VII) Final \\ approval of manuscript: All authors. \\ Correspondence to: Nicolas Kahn, MD. Department of Pneumology and Critical Care Medicine, Thoraxklinik, Heidelberg University Hospital, \\ Röntgenstr. 1, 69126 Heidelberg, Germany. Email: nicolas.kahn@med.uni-heidelberg.de.
}

\begin{abstract}
This review highlights a selection of abstracts from the 2017 ERS International Congress in Milan presented by the clinical assembly's groups of interventional pulmonology, general practice and primary care and rehabilitation and chronic care. Attention is brought to the increasing burden of disease of COPD and end-stage emphysema patients in China. The considerable efforts of Chinese pulmonary centers to conduct multicenter, randomized and controlled trials, investigating the possibilities of bronchoscopic lung volume reduction is underlined in the review. Furthermore, the key objectives of asthma management are stressed. We point out presentations emphasizing on achieving good control of daily symptoms and reducing the risk of asthma exacerbations. The findings of a randomized clinical trial evaluating the role of oxygen supplementation in rehabilitation therapy of COPD patients are analyzed. Additionally, another randomized clinical trial researching the influence of whole body vibration training (WBVT) in long-term (>1 year) lung transplant patients are discussed.
\end{abstract}

Keywords: Endoscopic lung volume reduction (ELVR); general practice, primary care; oxygen desaturation and supplementation; COPD; pulmonary rehabilitation

Submitted Aug 28, 2017. Accepted for publication Sep 22, 2017.

doi: $10.21037 /$ jtd.2017.11.43

View this article at: http://dx.doi.org/10.21037/jtd.2017.11.43

This short review highlights a selection of a few outstanding abstracts from the 2017 ERS International Congress in Milan (1). Only abstracts submitted to the clinical assembly (assembly 1) were considered for this publication.

Many interesting and novel findings are presented during the conference, which are not covered by this review. We hope to raise the interest of the readers of the fournal of Thoracic Diseases towards ERS activities and to join us at the ERS International Congress to share their newest scientific findings and discuss clinical challenges in the field of respiratory medicine.

\section{Interventional pulmonology}

Endoscopic lung volume reduction (ELVR) is a procedure using valves to occlude bronchi in emphysematous regions of the lung to allow for the expiration of air from the treated lobe of the lung. This technique is being adopted as a treatment option by an increasing number of pulmonary departments worldwide. This treatment option has been demonstrated to improve pulmonary function, exercise capacity and quality of life (QoL) in selected groups of patients.

In order to ensure clinical improvement and minimize 
possible complications for the patients, a careful selectionprocess is crucial (2). The most frequently used selection criteria include the low-attenuation area percentage (LAA\%), the heterogeneity score (HS) and fissure integrity (FI). One method of assessing collateral ventilation and to select candidates eligible for valve-based LVR is an invasive measurement with Chartis.

Schuhmann et al. have recently published a paper retrospectively comparing quantitative computed tomography (QCT) predictors of LVR outcome and compare the QCT model with Chartis in selecting likely responders to valve-based LVR treatment (3). In an abstract that will be presented at this year's conference they present data on a larger cohort of patients. They report to have compared three QCT predictors of response to Chartis measurement in 122 subjects. They conclude that QCT measurements of FI, HS and low-attenuation cluster slope correlate well with response to ELVR. The sensitivity (80\%) and specificity $(79 \%)$ of their model compares favourably to Chartis measurement (75\% accuracy in predicting lobar volume reduction). This opens a discussion on whether a combination of chartis measurement and QCT analysis may improve the total accuracy of ELVR response prediction.

In addition to patient selection prior to any intervention, an optimal method to objectively measure the efficacy of the procedure is discussed amongst experts in the field $(4,5)$. Besides improvement in forced expiratory volume (FEV1), reduction of residual volume (RV) and improvement in exercise capacity (6 min walk distance) a commonly used parameter is the target lobar volume reduction (TLVR). However, the currently used cut-off $(-350 \mathrm{~mL}$ TLVR) has until now not been satisfactorily, scientifically determined. Welling and colleagues submitted an abstract to the ERS annual conference 2017 presenting a new approach to address this problem. They used an anchor based method to define the minimal important difference (MID) for TLVR. FEV1, RV and SGRQ were used as anchors while TLVR was determined using QCT analysis by measuring the difference in target lobular volume between baseline and 6-month follow-up from a total of 318 severe emphysema patients from two different EBV trials. The combined MID (average of the three anchor-based MIDs) was $-563 \mathrm{~mL}$, or $-22.4 \%$ relative MID respectively.

The authors' approach of using an anchor-based method for three validated efficacy endpoints to establish a more robust TLVR cut-off in patients with severe emphysema will surely be useful for the interpretation of results from EBV-trials. It might also be a helpful tool for designing future ELVR trials.

The treatment of end-stage emphysema patients with valve-based ELVR is being implemented increasingly worldwide. Wang and colleagues will present the results of the first multicentre randomized controlled trial (RCT) conducted in China during the annual ERS conference in Milan 2017. The study was designed to assess the safety and efficacy of endobronchial valve treatment (Spiration Valve System, Olympus USA) following up the enrolled patients for 12 months.

A total of 12 Chinese pulmonary centres collaborated for this study enrolling a total of 101 subjects (66 treatments, 35 controls) with severe emphysema and complete fissures. Target lobe selection was based on visual HRCT only. Patients in the treatment arm underwent target lobe occlusion utilizing the Spiration Valve System while the control arm received optimal medical management. A significant and clinically meaningful improvement in FEV1 is reported with a response rate of $39.8 \%$ at 12 -month follow-up and a mean TLVR of $781 \mathrm{~mL}$. In addition, the authors report improvements of 6-minute walking distance (6MWD) as well as QoL measurements. Cases of late-serious adverse events were also documented in the treatment arm with mainly acute exacerbations $(n=6)$, lung abscess $(n=1)$ and pneumothorax. One patient deceased in the control group, while no fatalities were reported in the treatment arm.

Considering the burden of disease of COPD and the vast amount of end-stage emphysema patients in China it is highly commendable that considerable efforts are now being undertaken to conduct multicentre, randomized and controlled trials including Chinese pulmonary centres. This certainly increases the evidence on how patients should be selected for this procedure and the improvement that can be expected from it.

Overall we're excited about the new evidence presented in this field and discussions that will arise during the ERS annual conference and we hope to facilitate a vivid discussion on clinical decision making for the benefit of the patients affected.

\section{General practice and primary care}

Despite effective pharmacotherapy, asthma impairs the QoL in most patients (6). Psychological factors could be as important as physiological ones in perceiving symptoms and disease impact. That is why non-pharmacologic approaches are of great interest to patients. 
Thomas et al. investigated the usefulness of breathing retraining in 655 adult asthmatics in a 12-month, 3-armed RCT (7). They assessed a self-guided digital breathing retraining intervention delivered as DVD plus printed booklet (DVDB) versus three 'face-to-face' physiotherapist sessions plus booklet (FTF) (powered for equivalence), and with 'usual care' as control group (powered for superiority). The primary outcome in this study, AQLQ score, was significantly higher than control in the DVDB group (adjusted mean difference $0.28,95 \%$ CI: $0.11-0.44$, $\mathrm{P}<0.001)$. FTF was equivalent to DVDB $(0.04,95 \%$ CI: -0.16 to 0.24$)$ and number needed to treat $(\mathrm{NNT})$ was 8 in the DVDB group and 7 in FTF group. No significant differences in secondary outcomes were reported. Despite the better effect, healthcare costs were lower in both intervention groups than usual care, being lowest in the DVDB group. The authors conclude that breathing retraining programs improve QoL in patients with incompletely controlled asthma, reduce healthcare costs, but they do not have significant effect on lung function or airways inflammation. Breathing exercises could improve patient-reported outcomes and psychological state and they should be offered to all asthma patients with symptoms or impaired QoL despite standard treatment. However, the access to therapist who can provide this training may not be readily available everywhere (7).

On the other hand, QoL is significantly impaired in asthma exacerbation. Frequent exacerbations in children not only have an impact on QoL and school attendance but they are also associated with an accelerated loss of lung function. Guidelines emphasize monitoring should include objective assessment of asthma control (such as ACQ and ACT questionnaires) or morbidity scores (e.g., Royal College of Physicians three questions) as well as a future risk assessment of an exacerbation (5). A risk score for prednisolone use in adults is developed, however, no such score exists for children aged 5-12 years (8). With this justification, Buelo et al. tried to identify and weight factors associated with increased risk of exacerbation in children aged 5-12 years. Their systematic review included 48 papers from 6 databases, assessed by 2 independent reviewers. A meta-analysis was not performed due to heterogeneity. Four clinicians independently assessed each factor for both risk and confidence degree, based on study quality, effect sizes, biological plausibility and consistency of results. A consensus was achieved by discussion at a multidisciplinary workshop. A greatly increased risk of exacerbation was associated with presence of previous exacerbations and
African-American ethnicity (in US studies), a moderately/ greatly increased risk was present in patients with persistent symptoms, a moderately increased risk had patients with a sub-optimal drug regimen, high reliever use, co-morbid atopic diseases, allergic sensitization, poverty, exposure to tobacco smoke, and poor access to care, while a slightly increased risk was associated with younger age and obesity. The authors concluded that these clinical and demographic characteristics would help clinicians to 'spot the child' at increased risk and manage to reduce this risk. These data are particularly important due to fact that $5.1 \%$ of asthma deaths in one UK study are in children under the age of 10 years (9). Attention is drawn to population level factors (such as poverty and poor access to care) which may be used to target healthcare services and policy.

In conclusion, the key objective of the asthma management is to reduce the burden of disease by achieving good control of daily symptoms (QoL) and by reducing the risk of asthma exacerbations. Non-pharmacologic therapies may have benefit in patients with not optimal asthma control but they are underused due to difficulties in accessing therapy. Certain clinical and demographic features, which are readily available, could be used to detect increased risk in children. However, more studies are needed to assess whether or not these results are applicable to a broader group of patients.

\section{Rehabilitation and chronic care}

\section{Oxygen supplementation does not provide greater training} benefits in COPD than medical air: a RCT

Oxygen desaturation is frequently occurred in patients with COPD during exercise due to the imbalance between oxygen supply and oxygen demand, even in those without resting hypoxemia $(10,11)$. The decline of blood oxygen levels on exertion is attributed to respiratory, hemodynamic and peripheral muscle abnormalities or a combination thereof, and can lead to exercise intolerance and/or compromise the intensity of exercise training in COPD $(12,13)$ Evidences have also shown that oxygen administration during exercise improves performance, delays the onset of dynamic hyperinflation, mitigates dyspnea sensation, and doubles the effect of endurance training in pulmonary rehabilitation (PR) programs (14-16).

Alison et al. conducted the first multi-center, double-blind RCT which investigated the effectiveness of supplemental oxygen in optimizing training intensity and maximizing 
the benefits gained from PR compare to medical air (sham intervention) (17). They randomized 111 COPD patients, who did not receive long-term oxygen therapy (LTOT) and exhibited oxygen desaturation lower than $90 \%$ during the 6-minute walk test, into two groups; the oxygen group (OG) and the air group (AG). Both groups received the respective gas from a concentrator via nasal prongs at $5 \mathrm{~L} / \mathrm{min}$ during exercise training (3 times/week; 8 -week program) on treadmill and cycle ergometer. Investigators demonstrated similar improvement in exercise capacity and health-related QoL in both groups (all $\mathrm{P}<0.05$ for all outcomes) suggesting that supplemental oxygen does not provide greater training benefits compared to medical air in COPD patients with severe desaturation.

Previously, data from RCTs evaluated the effects of supplemental oxygen during exercise in $\mathrm{PR}$ program for COPD patients with exercise-induced oxygen desaturation, and significant differences amongst groups were not detected (18-20). Abernethy et al. also reported that there is no additional symptomatic benefit of oxygen over room air for relieving refractory dyspnea (21). Although these results can be debated according to the study of Emtner et al. (14), at least for the non-hypoxemic patients with COPD, the new findings from current largest rigorous RCT advocate for similar training benefits by the use of oxygen or medical air.

\section{Whole body vibration training (WBVT) in long-term (>1 year) lung transplant patients-a RCT}

WBVT is an exercise modality inducing myoelectric stimulation by oscillatory vibrations to a subject standing on a vibrating platform (22). It is an efficacious mode of exercise training in patients with COPD that improves exercise capacity and lower limbs functional performance (23-25). WBVT is also associated with increased irisin levels as marker of muscle activity in COPD and may improve health-related QoL (26).

Gloeckl et al. have demonstrated the effectiveness of complementary WBVT as a component of PR on the outcomes of exercise capacity in lung transplant patients (LTx) for COPD, who underwent lung-transplantation before less than 1-year and described as "short-term" LTxrecipients (27). Recently, same investigators published new data of a RCT regarding the beneficial effects of complementary WBVT in the "long-term" LTx patients ( $>1$-year transplanted) enrolled in a 4-week PR program. They randomized 70 long-term LTx patients for COPD or interstitial lung disease (ILD) into two groups based to the exercise training performed by patients. In the first group, patients performed squat training on the vibrating platform (WBVT group: squat training combined with WBVT). In the second group, patients performed ground-based squat training (control group: dynamic squat exercises on the floor). Investigators demonstrated that patients assigned to complementary WBVT as an add-on exercise modality, increased the 6MWD (WBVT: $44 \pm 44 \mathrm{~m}$ vs. controls: $22 \pm 34 \mathrm{~m}$, $\mathrm{P}<0.027$ ), and the peak cycling work rate (WBVT: $15 \pm 9$ vs. $10 \pm 9 \mathrm{~W}, \mathrm{P}=0.044)$ superior compared to control group. Moreover, an increase of insulin-like growth factor $(+18 \pm 49$ $\mathrm{ng} / \mathrm{mL})$ in WBVT group was reported $(\mathrm{P}=0.035)$.

The pre-transplant condition in COPD is associated with poor muscle mass (28), muscle fiber atrophy (29), and low proportion of muscle fiber type I (30), followed by muscle weakness (31), and low muscle endurance (32). After lung-transplantation, further worsening of skeletal muscle is likely to be occurred as a consequence of immunosuppressant medications (33). For instance, steroidassociated myopathy is related to muscular atrophy, which can be due to suppressed protein synthesis and growth, enhanced proteolysis, and apoptosis induction, therefore affecting the muscle condition of long-term LTx patients (34). Consequently, neuromuscular adaptations including improvements in balance and muscle power as positive effects of WBVT (35) could be highly beneficial to lungtransplanted patients. The new findings of current RCT promote the use of WBVT in combination to conventional exercise training in LTx patients assigned to PR.

\section{Acknowledgements}

Funding: Vasileios Andrianopoulos is the recipient of an ERS-EU RESPIRE2 Marie Sklodowska-Curie Postdoctoral Research Fellowship-Number MCF (8465)-2015.

\section{Footnote}

Conflicts of Interest: The authors have no conflicts of interest to declare.

\section{References}

1. ERS International Congress 2017. Eur Respir J 2017;50:Suppl 61 (abstract).

2. Eberhardt R, Gompelmann D, Herth FJ, et al. Endoscopic bronchial valve treatment: patient selection and special 
considerations. Int J Chron Obstruct Pulmon Dis 2015;10:2147-57.

3. Schuhmann M, Raffy P, Yin Y, et al. Computed tomography predictors of response to endobronchial valve lung reduction treatment. Comparison with Chartis. Am J Respir Crit Care Med 2015;191:767-74.

4. Koster TD, van Rikxoort EM, Huebner RH, et al. Predicting Lung Volume Reduction after Endobronchial Valve Therapy Is Maximized Using a Combination of Diagnostic Tools. Respiration 2016;92:150-7.

5. Slebos DJ, Shah PL, Herth FJ, et al. Endobronchial Valves for Endoscopic Lung Volume Reduction: Best Practice Recommendations from Expert Panel on Endoscopic Lung Volume Reduction. Respiration 2017;93:138-50.

6. Global Initiative for Asthma (GINA). The Global Strategy for Asthma Management and Prevention. GINA, 2017. Available online: http://ginasthma.org/2017-gina-reportglobal-strategy-for-asthma-management-and-prevention/

7. Thomas M, Bruton A. Breathing exercises for asthma. Breathe 2014;10:312-22.

8. Blakey JD, Obediat M, Pogson Z, et al. A Simple Asthma Severity Score Predicts Exacerbations. Am J Respir Crit Care Med 2011;183:A2248.

9. Royal College of Physicians. Why Asthma Still Kills: the National Review of Asthma Deaths (NRAD) Confidential Enquiry Report. London, RCP, 2014. Available online: www.rcplondon.ac.uk/sites/default/files/why-asthma-stillkills-full-report.pdf

10. Andrianopoulos V, Franssen FM, Peeters JP, et al. Exercise-induced oxygen desaturation in COPD patients without resting hypoxemia. Respir Physiol Neurobiol 2014;190:40-6.

11. van Gestel AJ, Clarenbach CF, Stöwhas AC, et al. Prevalence and prediction of exercise-induced oxygen desaturation in patients with chronic obstructive pulmonary disease. Respiration 2012;84:353-9.

12. Vogiatzis I, Zakynthinos G, Andrianopoulos V. Mechanisms of physical activity limitation in chronic lung diseases. Pulm Med 2012;2012:634761.

13. Spruit MA, Singh SJ, Garvey C, et al. An official American Thoracic Society/European Respiratory Society statement: key concepts and advances in pulmonary rehabilitation. Am J Respir Crit Care Med 2013;188:e13-64.

14. Emtner M, Porszasz J, Burns M, et al. Benefits of supplemental oxygen in exercise training in nonhypoxemic chronic obstructive pulmonary disease patients. Am J Respir Crit Care Med 2003;168:1034-42.

15. Vesteng LK, Skjørten I, Melsom M, et al. COPD and oxygen supplementation: Improvement in inspiratory capacity and dyspnea during exercise. Eur Respir J 2015;46:PA4603.

16. Neunhäuserer D, Steidle-Kloc E, Weiss G, et al. Supplemental Oxygen During High-Intensity Exercise Training in Nonhypoxemic Chronic Obstructive Pulmonary Disease. Am J Med 2016;129:1185-93.

17. Alison JA, McKeough ZJ, Jenkins SC, et al. A randomised controlled trial of supplemental oxygen versus medical air during exercise training in people with chronic obstructive pulmonary disease: supplemental oxygen in pulmonary rehabilitation trial (SuppORT) (Protocol). BMC Pulm Med 2016;16:25.

18. Garrod R, Paul EA, Wedzicha JA. Supplemental oxygen during pulmonary rehabilitation in patients with COPD with exercise hypoxaemia. Thorax 2000;55:539-43.

19. Rooyackers JM, Dekhuijzen PN, Van Herwaarden CL, et al. Training with supplemental oxygen in patients with COPD and hypoxaemia at peak exercise. Eur Respir J 1997;10:1278-84.

20. Wadell K, Henriksson-Larsén K, Lundgren R. Physical training with and without oxygen in patients with chronic obstructive pulmonary disease and exercise-induced hypoxaemia. J Rehabil Med 2001;33:200-5.

21. Abernethy AP, McDonald CF, Frith PA, et al. Effect of palliative oxygen versus room air in relief of breathlessness in patients with refractory dyspnoea: a double-blind, randomised controlled trial. Lancet 2010;376:784-93.

22. Gloeckl R, Heinzelmann I, Kenn K. Whole body vibration training in patients with COPD: A systematic review. Chron Respir Dis 2015;12:212-21.

23. Gloeckl R, Heinzelmann I, Baeuerle S, et al. Effects of whole body vibration in patients with chronic obstructive pulmonary disease--a randomized controlled trial. Respir Med 2012;106:75-83.

24. Pleguezuelos E, Pérez ME, Guirao L, et al. Effects of whole body vibration training in patients with severe chronic obstructive pulmonary disease. Respirology 2013;18:1028-34.

25. Furness T, Joseph C, Naughton G, et al. Benefits of wholebody vibration to people with COPD: a community-based efficacy trial. BMC Pulm Med 2014;14:38.

26. Greulich T, Nell C, Koepke J, et al. Benefits of whole body vibration training in patients hospitalised for COPD exacerbations - a randomized clinical trial. BMC Pulm Med 2014;14:60.

27. Gloeckl R, Heinzelmann I, Seeberg S, et al. Effects of complementary whole-body vibration training in patients 
after lung transplantation: A randomized, controlled trial. J Heart Lung Transplant 2015;34:1455-61.

28. Bernard S, LeBlanc P, Whittom F, et al. Peripheral muscle weakness in patients with chronic obstructive pulmonary disease. Am J Respir Crit Care Med 1998;158:629-34.

29. Whittom F, Jobin J, Simard PM, et al. Histochemical and morphological characteristics of the vastus lateralis muscle in patients with chronic obstructive pulmonary disease. Med Sci Sports Exerc 1998;30:1467-74.

30. Vogiatzis I, Terzis G, Stratakos G, et al. Effect of pulmonary rehabilitation on peripheral muscle fiber remodeling in patients with COPD in GOLD stages II to IV. Chest 2011;140:744-52.

31. Gosselink R, Troosters T, Decramer M. Distribution of muscle weakness in patients with stable chronic obstructive pulmonary disease. J Cardiopulm Rehabil 2000;20:353-60.

32. Allaire J, Maltais F, Doyon JF, et al. Peripheral muscle endurance and the oxidative profile of the quadriceps in patients with COPD. Thorax 2004;59:673-8.

33. Mathur S, Levy RD, Reid WD. Skeletal muscle strength and endurance in recipients of lung transplants. Cardiopulm Phys Ther J 2008;19:84-93.

34. Valiyil R, Christopher-Stine L. Drug-related myopathies of which the clinician should be aware. Curr Rheumatol Rep 2010;12:213-20.

35. Gloeckl R, Jarosch I, Bengsch U, et al. What's the secret behind the benefits of whole-body vibration training in patients with COPD? A randomized, controlled trial. Respir Med 2017;126:17-24.
Cite this article as: Kahn N, Andrianopoulos V, Mekov E, Herth FJ. ERS International Congress 2017: a peek of outstanding abstracts from the clinical assembly. J Thorac Dis 2017;9(Suppl 16):S1526-S1531. doi: 10.21037/jtd.2017.11.43 